\title{
ESTUDO CRÍTICO SOBRE UTILIZAÇÃO DE FITOTERÁPICOS POR PRATICANTES DE EXERCÍCIO FÍSICO EM ACADEMIAS DE MUSCULAÇÃO
}

\section{Bruno Esteves Conde}

Doutorando em Ecologia/Universidade Federal de Juiz de Fora

bcondebio@hotmail.com

\author{
Arthur Ladeira Macedo \\ Doutorando em Química (UFF) \\ arthur_macedo@id.uff.br \\ Andrea Esteves Martins \\ Mestranda em Ecologia/Universidade Federal de Juiz de Fora \\ aembiol@hotmail.com
}

Amanda Surerus Fonseca

Graduanda em Ciências Biológicas/Universidade Federal de Ouro Preto

mandinhasurerus@gmail.com

\author{
Aline Moreira de Siqueira \\ Mestre em Ecologia/Universidade Federal de Juiz de Fora \\ alinemds1@yahoo.com.br
}

Gabriel Hiotti Lino de Souza

Graduando em Engenharia Ambiental/ Faculdade Doctum

gabrielhiotti14@hotmail.com

\section{Izabela Taiana Salazar Rogério}

Mestre em Ecologia Aplicada a Conservação da Biodiversidade/ Universidade Federal de Juiz de Fora izasalazar88@gmail.com

\section{RESUMO}

Considerando-se a crescente demanda da utilização de fitoterápicos em academias de musculação, o presente estudo objetivou avaliar a forma de obtenção de informações sobre os fitoterápicos e os riscos causados por tais usos em academias de musculação. Aplicaram-se formulários estruturados à praticantes de atividades físicas de duas academias de ginástica de Juiz de Fora/MG. Realizou-se pesquisa em literatura científica sobre as espécies utilizadas. $O$ principal objetivo para o uso de fitoterápicos ergogênicos foi $o$ emagrecimento e a principal alegação para o uso foi melhoria no desempenho para a realização da atividade física praticada. Encontrou-se que Camellia sinensis, Baccharis trimera, Paullinia cupana, Phaseoulus vulgaris e Gymnema sylvestre vem sendo usados de maneira consonante com a literatura científica. Citrus aurantium apresentou atividade comprovada, porém vários artigos discutem sua toxicidade. Tribulus terrestris apresentou-se contraditório, uma vez que alguns artigos comprovam sua eficácia e outros comprovam sua incapacidade de exercer efeitos significativos. Não foram encontrados artigos que discutem a atividade citada para Oenothera biennis e Cynara scolymus. Nota-se que mesmo a legislação estando de acordo com a realidade dos profissionais de saúde, esta não é eficiente em garantir o uso respaldado por médicos, nutricionistas e farmacêuticos no âmbito de academias de musculação.

Palavras-Chave: Farmacologia; Performance Física; Planta Medicinal. 


\section{ABSTRACT}

Considering the growing demand of the use of herbal medicines at gyms, the present study aimed at evaluating the way of obtaining information on phytotherapeutic agents and the risks caused by such uses in gyms. Structured forms were applied to physical activity practitioners in two gyms of Juiz de Fora/MG, Brazil. Research in scientific literature was conducted for the used species. The main purpose for the use of ergogenic phytoterapics was weight loss and the main claim for the use was improvement in the performance for holding the practiced physical activity in the practiced physical activity. Camellia sinensis, Baccharis trimera, Paullinia cupana, Phaseoulus vulgaris e Gymnema sylvestre are being used in a way consonant with the scientific literature. Citrus aurantium had proven activity, but many articles discuss its toxicity. Tribulus terrestris showed contradictory results, since some articles proved its efficacy and others have demonstrated its inability to have significant effects. Articles that discuss the mentioned activity of Oenothera biennis e Cynara scolymus are scanty. It was noted that even the legislation is in accordance with the reality of health professionals, this is not effective in ensuring the use supported by physicians, nutritionists and pharmacists in the context of gyms.

Key words: Medicinal Plant; Popular Knowledge; Pharmacology; Physical Performance.

\section{INTRODUÇÃO}

A busca pelo modelo ideal de corpo perfeito e que vem sendo estabelecido principalmente pela mídia é crescente, fazendo com que inúmeras pessoas acabam ultrapassando suas limites com relação à própria estrutura física, na tentativa da obtenção de uma aparência estética que julgam ser mais importante que o cuidado com a própria saúde (CASTRO, 2010). Este referido modelo estético e a falta de uma cultura corporal saudável têm levado a população a usar de forma abusiva substâncias tidas como promotoras de melhorias estéticas (SANTOS; SANTOS, 2002). Dentre estas substâncias, destacam-se os fitoterápicos, medicamentos à base de plantas, tidos popularmente como menos agressivos à saúde (YUNES; PEDROSA; FILHO, 2001). Entretanto muito destes fitoterápicos são utilizados com base em informações não científicas como fóruns de discussão na internet, blogs e também na oralidade que ocorre principalmente no contexto das acadêmicas de ginástica (RATES, 2001).

São muitos os efeitos prometidos pelos fabricantes dos produtos fitoterápicos, tais como, a melhoria da capacidade de trabalho, a eliminação do cansaço mental, a melhoria do desempenho físico dentre outros (TIRAPEGUI, 2005). Tais substâncias capazes de produzir esses tipos de efeitos são chamadas de substâncias ergogênicas (FOX; BOWERS; FOSS, 1988).

Muitas destas substâncias ditas leigamente como ergogênicas produzem efeitos diretamente contrários, e assim sendo consideradas como substâncias ergolíticas e acabam por interferir negativamente no rendimento de quem as utiliza (KENNY; WILMORE; COSTILL, 2011). Sendo assim a ganância por obter um ótimo desempenho numa atividade física e um corpo idealizado pela sociedade fez com que o conceito de "Esporte e Saúde" entrasse em detrimento uma vez que apenas a estética seja enfatizada (BARRETO, 2003).

Tendo em vista a demanda crescente da utilização de fitoterápicos em academias de musculação (KLEIN et al., 2009) nota-se que a prescrição por profissionais capacitados é de fundamental importância para a manutenção da saúde e da qualidade de vida, devendo ter como base a comprovação científica e dosagens específicas individuais. Segundo a legislação brasileira, são profissionais capacitados para prescrever fitoterápicos comumente usados em academias os médicos (CFM, 1992), nutricionistas (CFN, 2013), além do farmacêutico, que formalmente não é um profissional prescritor, mas que pode indicar o uso de fitoterápicos isentos de prescrição (CFF, 2011).

O presente estudo teve como objetivo o levantamento da utilização de fitoterápicos ergogênicos em duas academias de musculação da cidade de Juiz de Fora, bem como a finalidade do uso e o meio pelo qual foi prescrito tal medicamento, com posterior análise crítica do uso com base em literatura científica, a fim de avaliar a interação do sistema formal de saúde no contexto das academias de musculação e os riscos causados pela utilização indiscriminada de tais medicamentos. 


\section{METODOLOGIA}

Para a coleta de dados utilizou-se método similar ao de Santos e Santos (2002), em que foi amostrado um total de 5\% (100 pessoas) por seleção aleatória, do total de alunos matriculados na modalidade musculação de cada uma das academias, sendo $50 \%$ do sexo masculino e $50 \%$ do sexo feminino.

Foram aplicados formulários estruturados (ALEXÍADES, 1996) que abordaram dados como: 1) idade; 2) tempo de academia; 3) objetivo almejado pela musculação; 4) se já utilizou e/ou utiliza de fitoterápicos com propósito ergogênico e quais os termos referidos para os fitoterápicos utilizados, bem como o nome da farmácia para uma posterior avaliação do nome científico da espécie botânica utilizada como matéria prima; 5) para qual finalidade os utilizam; 6) quem prescreveu/indicou o medicamento; 7) caso não tenha sido prescrito por um médico, quando vai a um médico informa o uso do mesmo e qual opinião dele.

Com os dados referentes ao uso principal, posologia e duração do tratamento com os referidos fitoterápicos, realizou-se pesquisa, a fim de comprovar a eficácia e ou possível toxidez, com base na busca de literaturas científicas contidas nos bancos de dados: Scopus; Chemical Abstracts; International Pharmaceutical Abstracts; Literatura Latino-Americana e do Caribe em Ciências da Saúde; Analytical Abstracs; OLDMEDLINE e PUBMED.

\section{RESULTADOS}

A partir da análise dos dados foi possível constatar que entre os 100 entrevistados a idade média foi de 35 anos e o tempo médio de academia é de 3 anos e 4 meses. O principal objetivo almejado por tais praticantes é estética com $75 \%$ (Figura 1).

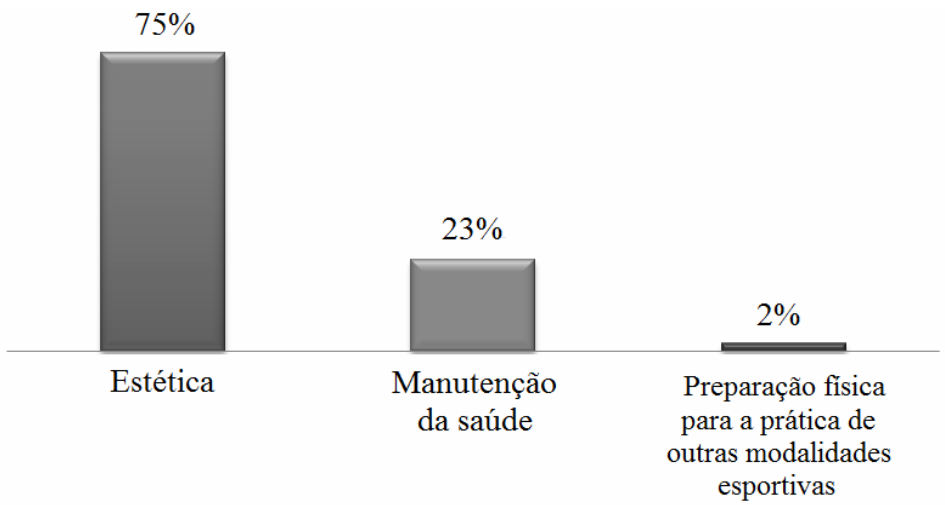

Figura 1: Principais objetivos almejados pelos participantes a partir a realização de atividades físicas no contexto de academias de ginástica de Juiz de Fora, MG.

Em relação aos participantes que alegaram utilizar ou já terem utilizado fitoterápicos, pode-se notar que a maioria respondeu positivamente (85\%) (Figura 2). 


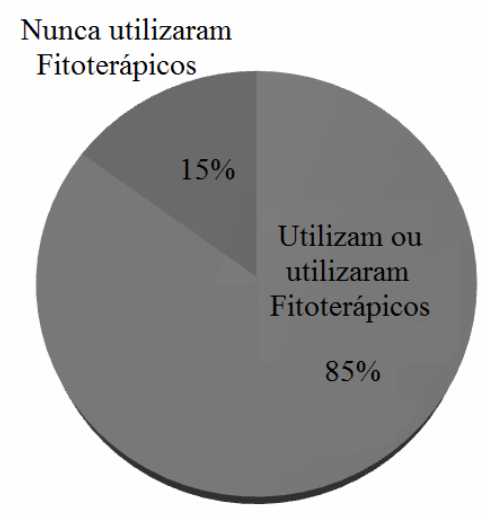

Figura 2: Utilização de fitoterápicos pelos entrevistados

Foram levantados 16 termos vernaculares quanto aos nomes dos fitoterápicos, dos quais foi possível identificar, a partir da conferência do produto junto à farmácia e informações adquiridas com revendedores, nove espécies (Tabela 1) com seus respectivos usos principais e percentuais de utilização. Os nomes científicos foram confirmados quanto à atualização dos nomes botânicos junto ao site oficial do Missouri Botanical Garden.

Tabela 1: Nomes vernaculares dos fitoterápicos e as referidas espécies utilizadas como matéria prima com respectivos percentuais de uso dentre os 100 entrevistados.

\begin{tabular}{|l|l|l|c|}
\hline \multicolumn{1}{|c|}{$\begin{array}{c}\text { NOME } \\
\text { VERNACULAR }\end{array}$} & \multicolumn{1}{c|}{ ESPÉCIE PRINCIPAL } & \multicolumn{1}{c|}{ USO PR } \\
\hline Chá verde & Camellia sinensis (L.) Kuntze & Emagrecer & 61 \\
\hline Tribulos & Tribulus terrestris L. & Aumento da força e da massa muscular & 60 \\
\hline Carqueja & Baccharis trimera (Less.) DC. & Emagrecer & 19 \\
\hline Guaraná & Paullinia cupana Kunth & Emagrecer e aumento de força & 15 \\
\hline Óleo de Prímula & Oenothera biennis L. & Emagrecer & 14 \\
\hline Cavalinha & Equisetum arvense L. & Emagrecer & 14 \\
\hline Alcachofra & Cynara scolymus L. & Aumento de massa muscular & 9 \\
\hline Citrus aurantio & Citrus aurantium L. & Emagrecer & 8 \\
\hline Giminema & Gymnema sylvestre (Retz.) R. Br. & Emagrecer & 2 \\
\hline
\end{tabular}




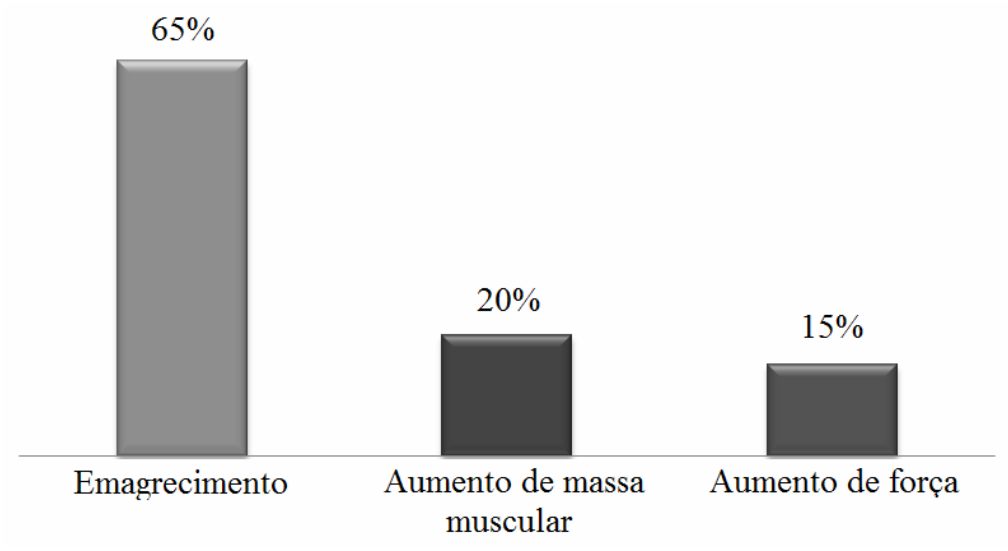

Figura 3: Objetivos almejados pelos entrevistados

Quanto aos objetivos almejados pela utilização de fitoterápicos, destaca-se a categoria emagrecimento (Figura 3), com vista da estética e da melhoria da qualidade de vida.

Dentre os que recomendaram o uso de fitoterápicos predominaram professores das próprias academias, colegas de academia e pesquisas em internet (Figura 4).

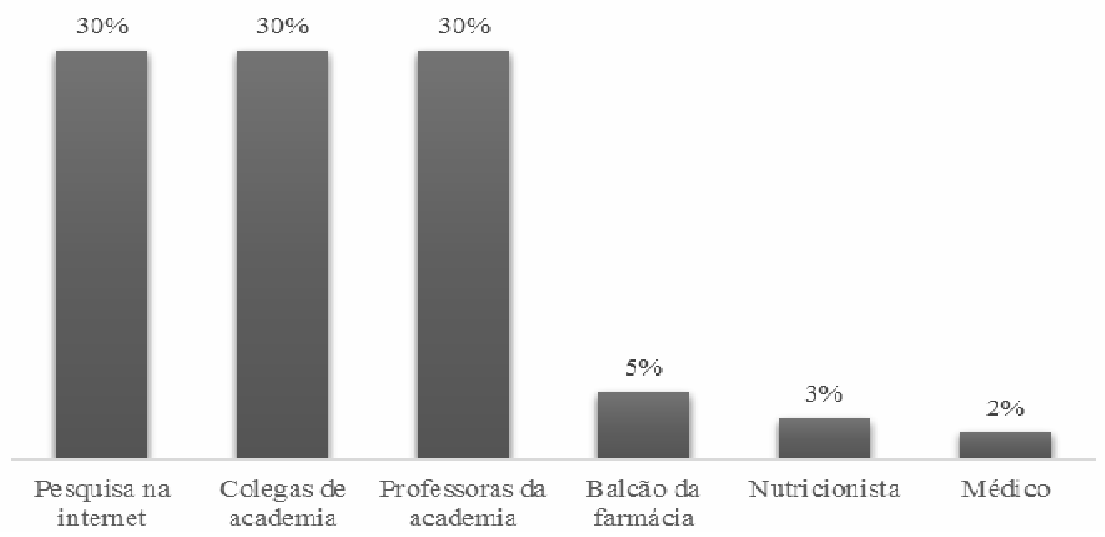

Figura 4: Meios pelo qual os entrevistados vieram a adquirir indicação do uso de fitoterápicos.

Dentre os que relataram sobre o uso de fitoterápicos, apenas $8 \%$ informaram aos médicos (Figura 5), sendo que, na maioria das vezes $(80 \%)$ a opinião dos mesmos foi indiferente segundo os usuários entrevistados (Figura 6). 


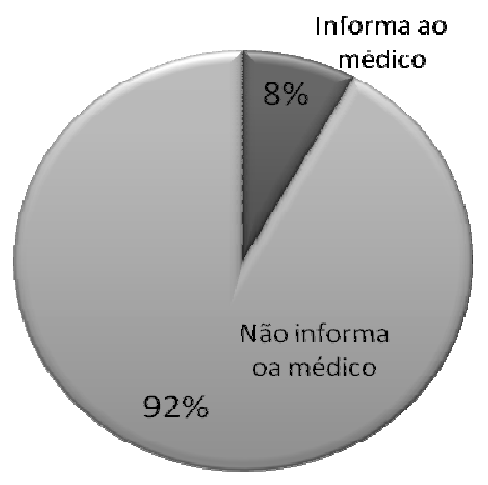

Figura 5: Percentual de entrevistados que informam aos médicos sobre o uso de fitoterápicos prescritos por pessoas não qualificadas.

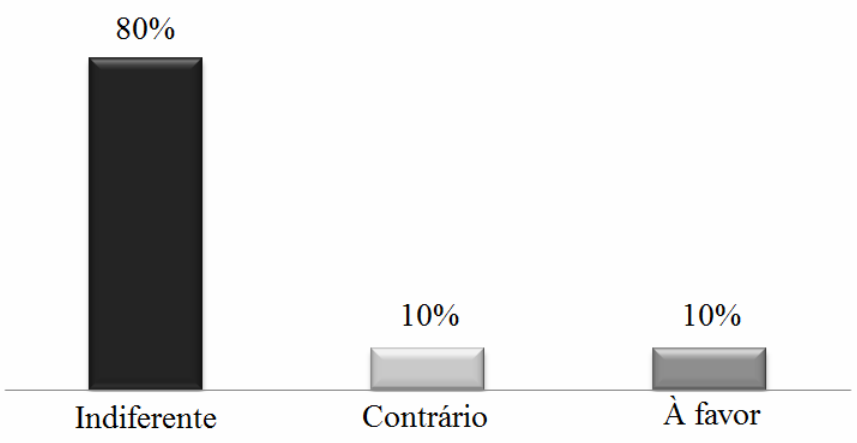

Figura 6: Opinião dos médicos, segundo os usuários entrevistados, quanto ao uso de fitoterápicos dos participantes que relataram utilizar prescrição por não profissional de saúde qualificado.

Quanto à comparação dos usos relatados pelos usuários de fitoterápicos com a literatura científica, apenas $O$. biennes e C. scolymus não apresentaram nenhum estudo que confirmasse diretamente o uso principal (Tabela 2). Dentre os que tiveram o uso principal confirmado, $T$. terrestris apresentou resultados divergentes e $C$. aurantium apresentou várias descrições de toxicidade. 
Quadro 1: Comparação entre os usos principais dos fitoterápicos e suas respectivas confirmações farmacológicas baseadas em revisão de literatura científica em bancos de dados especializados

\begin{tabular}{|c|c|c|}
\hline ESPÈCIE & USO PRINCIPAL & $\begin{array}{l}\text { CONFIRMAÇÃO EM } \\
\text { LITERATURA } \\
\text { CIENTÍFICA }\end{array}$ \\
\hline Camellia sinensis (L.) Kuntze & Emagrecer & $\begin{array}{l}\text { (SAE-TAN; ROGERS; } \\
\text { LAMBERT, 2015; } \\
\text { TORRES-FUENTES et al., } \\
\text { 2015; XU et al., 2015; } \\
\text { YANG et al., 2014) }\end{array}$ \\
\hline Tribulus terrestris $\mathrm{L}$. & Aumento da força e da massa muscular & $\begin{array}{l}\text { (BROWN et al., 2001; } \\
\text { MILASIUS; DADELIENE; } \\
\text { SKERNEVICIUS, 2009) }\end{array}$ \\
\hline Baccharis trimera (Less.) DC. & Emagrecer & $\begin{array}{l}\text { (CIFUENTE et al., 2010; } \\
\text { SOUZA et al., 2011; } \\
\text { SOUZA et al., 2012) }\end{array}$ \\
\hline Paullinia cupana Kunth & Emagrecer e aumento de força & $\begin{array}{l}\text { (ESPINOLA et al., 1997; } \\
\text { LIMA et al., 2005) }\end{array}$ \\
\hline Oenothera biennis L. & Emagrecer & - \\
\hline Phaseolus vulgaris L. & Emagrecer & $\begin{array}{l}\text { (LOI et al., 2013a;2013b; } \\
\text { LU et al., 2012; } \\
\text { RONDANELLI et al., 2011; } \\
\text { ZHU; JIANG; THOMPSON, } \\
\text { 2012) }\end{array}$ \\
\hline Cynara scolymus L. & Aumento de massa muscular & - \\
\hline Citrus aurantium $\mathrm{L}$. & Emagrecer & $\begin{array}{l}\text { (GRAY; WOOLF, 2005; } \\
\text { HONG et al., 2012; STOHS; } \\
\text { PREUSS; SHARA, 2012) }\end{array}$ \\
\hline Gymnema sylvestre (Retz.) R. Br. & Emagrecer & $\begin{array}{l}\text { (KUMAR et al., 2012;2013; } \\
\text { POTHURAJU et al., 2014) }\end{array}$ \\
\hline
\end{tabular}

\section{DISCUSSÃ̃O}

Pode-se relacionar a grande porcentagem $(75 \%)$ de praticantes de atividade física que responderam "estética" como o principal objetivo almejado, ao aumento pela busca da padronização estética vinculado à autoestima, sucesso, jovialidade e aceitabilidade social, como descrito por Costa e Venâncio (2004).

A porcentagem de utilização de fitoterápicos coincide com os dados estimados mundialmente (OMS, 2002) sobre a utilização de fitoterápicos para tratamentos de saúde (Figura 2). A utilização de fitoterápicos é cada vez mais frequente e se configura como uma alternativa para diversos tipos de tratamentos, dentre eles para o emagrecimento (VALENZUELA, 2004).

O emagrecimento foi relatado como principal objetivo para a utilização de fitoterápicos. No contexto atual, ter um corpo magro é estar nos padrões sociais de beleza (CAVICHIOLI; ABOURIHAN; PASSONI, 2012) o que pode justificar tal dado. Além disso, Lee et al. (2015) demonstrou que estar fora dos padrões sociais de beleza pode gerar discriminação em processos seletivos de empregos, o reforça a "necessidade" de 
obter resultados rápidos e satisfatórios com dietas e exercício físico. Para isso, muitas pessoas recorrem ao uso de medicamentos e/ou suplementos (MAIOLI, 2012). No presente trabalho foram citados nove fitoterápicos pelos entrevistados.

Dentre os fitoterápicos citados, $C$. sinensis apresenta uma boa fundamentação para ajudar no emagrecimento. Yang et al. (2014) demonstraram que o extrato desta planta reduz a adipogênese em humanos. Xu et al. (2015) demonstraram que os polifenóis, polissacarídeos e a cafeína presentes nesta espécie reduzem a concentração de leptina no soro e inibem a absorção de ácidos graxos em camundongos. Sae-Tan et al. (2015) encontraram que a associação de do chá de $C$. sinensis descafeinado e exercícios físicos induz a transformação de tecido adiposo branco em tecido adiposo marrom semelhante à gordura em ratos.

Os estudos sobre T. terrestris apresentaram-se contraditórios. Milasius, Dadeliene e Skernevivius (2009) demonstraram que atletas que consumiram um suplemento a base desta espécie três vezes por dia por 20 dias apresentaram maior potência muscular anaeróbia alática e maior glicólise anaeróbia alática e Brown et al. (2001), ao analisarem a ingestão de um composto a base de T. terrestris, encontram um aumento na concentração de testosterona livre no sangue. Por outro lado, Rogerson et al. (2007) encontraram em sua pesquisa que o consumo de $T$. terrestris uma vez por dia durante 28 dias não é capaz de aumentar significativamente a força ou a massa muscular de atletas de rugby. Além disso, os resultados encontrados por Brown et al. (2000) vão de encontro aos de Brown et al. (2001), uma vez que o mesmo composto foi testado e não foi capaz de produzir aumento de testosterona e nem capaz de promover adaptação em treinos de resistência. Antonio et al. (2000) demonstraram que a suplementação alimentar com T. terrestris não alterou a composição corporal nem aumentou a performance em exercícios de homens treinados para resistência. Um relato de caso recente sobre um homem de 30 anos, Ryan et al. (2015) apresentaram evidências de nefrotoxicidade e aumento na produção de bílis pela ingestão de $T$. terrestris.

Souza et al. (2011) sugeriram que $B$. trimera pode ser utilizada como coadjuvante no processo de emagrecimento, uma vez que apresenta atividade contra lipase pancreática e $\alpha$ e $\beta$ glicosidases in vitro, além de possuir antinutrientes como saponinas e polifenóis. Foi demonstrado também que esta espécie possui atividade colerética (CIFUENTE et al., 2010) e antidiabética não associada à perda de peso em camundongos (OLIVEIRA et al., 2005). É importante ressaltar que extratos de B. trimera apresentaram nefro e hepatotoxicidade em ratas prenhas, sendo um indício de que deve-se ter ainda mais atenção no uso em humanos durante o período de gestação (GRANCE et al., 2008).

A utilização de $P$. cupana apresenta bom respaldo na literatura científica. Um estudo conduzido em ratos em camundongos demonstrou que uma suspensão de baixa concentração foi capaz aumentar a capacidade física dos animais (ESPINOLA et al., 1997). Os autores também demonstraram que doses elevadas e o uso de cafeína isolada não foram capazes de produzir o mesmo efeito. Lima et al. (2005) evidenciaram que o extrato de $P$. cupana aumentou o metabolismo de lipídeos em ratos e que este efeito está associado à presença da cafeína. Quanto à efeitos psicológicos do uso, Silvestrini, Mariano e Cosentino (2013) demonstraram que $350 \mathrm{mg}$ administradas três vezes por dia por cinco dias consecutivos não é capaz de alterar as escalas de ansiedade, bem estar e de humor, porém em um relato de caso de uma paciente de 38 anos indica a possibilidade de atrofia do lobo frontal direito do cérebro após uma superdosagem (PENDLETON et al., 2012). Esta condição foi revertida após a descontinuação do uso.

Não foram encontrados artigos que relacionassem diretamente $O$. biennis à perda de peso. Um estudo clínico demonstrou que a combinação de policlonasol, extrato de tomate, procianidinas da uva e óleo de $O$. biennis é eficaz e seguro como hipocolesterolêmico por reduzir o LDL e o colesterol total, além de modular os índices de oxidação do organismo em curto prazo (GUPTA et al., 2012). Porém são necessários estudos utilizando somente $O$. biennis para a confirmação da atividade desta espécie.

O extrato de $P$. vulgaris foi indicado como potencial para o tratamento de obesidade, para a redução do desejo por alimentos e consequentemente para excessos alimentares por ter apresentado estes efeitos em ratos e camundongos (LOI et al., 2013b). Loi et al. (2013a) também demonstraram estas atividades, porém evidenciaram que a associação de $P$. vulgaris e $C$. scolymus é mais interessante visto que além dos efeitos anorexígenos de $P$. vulgaris apresenta um efeito aditivo de ambas as plantas na redução da glicemia pósprandial em camundongos, estes efeitos também foram detectados em estudo clínico (RONDANELLI et al., 2011). O extrato desta planta também apresentou capacidade de aumentar as taxas de HDL em humanos adultos e obesos (LU et al., 2012). O consumo de P. vulgaris seco apresentou atividade na manutenção do 
peso normal em ratos e camundongos com dieta hipercalórica, além de modular os fatores de risco cardiovascular (ZHU et al., 2012).

$\mathrm{Na}$ revisão realizada, não foram encontrados estudos que abordassem o ganho de massa muscular pela utilização de C. scolymus, porém, como descrito anteriormente, seu uso em associação com $P$. vulgaris na manutenção e perda e de peso é descrito. Embora não tenha sido encontrada uma relação direta com o ganho de massa magra, a utilização concomitante de $C$. scolymus a creatina reduziu os efeitos indesejados da creatina, como o aumento da ureia, creatinina, sódio, amônia e lactato no plasma em ratos (AHMED; MANNAA; EL-SAYED, 2008).

Em extensiva revisão bibliográfica, Bent, Padula e Neuhaus (2004) encontraram apenas um estudo clínico que avaliava o uso de $C$. aurantium para perda de peso, porém este não apresentava diferença estatística do controle. Esta planta apresenta aminas com efeito adrenérgico como $m$-sinefrina, tiramina e octopamina que exercem ação indireta na lipólise em humanos, mas também são responsáveis por vários efeitos adversos como aumento da ansiedade, sudorese, taquicardia e hipertensão (GRAY; WOOLF, 2005). Hansen et al. (2012), em estudo pré-clínico, demonstraram que a sinefrina causa aumento da pressão arterial e ritmo cardíaco tanto quando administrada isoladamente quanto em mistura no extrato, sendo o segundo caso mais pronunciado. Foi demonstrado em estudo in vitro que a $p$-sinefrina presente no extrato de $C$. aurantium aumenta o consumo basal de glicose induzido por insulina (HONG et al., 2012), porém os autores detectaram também o aumento da produção de ácido lático. Stohs, Preuss e Shara (2012) após uma longa revisão de literatura sobre ensaios clínicos que avaliaram $C$. aurantium concluíram que ela é efetiva na perda de peso não apresenta riscos cardíacos. Embora estes autores tenham chegado à conclusão de que $C$. aurantium é segura, com base nos artigos citados anteriormente, não aconselha-se que a utilização deste fitoterápico por pacientes que apresentem problemas cardíacos. Além disso, Schmitt et al. (2012) demonstraram que a associação de $p$-sinefrina, efedrina, salicina e cafeína é tóxica para camundongos, podendo levar a efeitos como convulsão, salivação, agitação e hemorragia cardiopulmonar.

Estudos pré-clínicos demonstraram que extratos de G. sylvestre reduziram significantemente os lipídeos, leptina, insulina, glicose, apolipoproteína-B e LDL séricos além de aumentar o HDL, apolipoproteína-A1 e enzimas antioxidantes do fígado, indicando que os extratos possuem efeito antiobesidade (KUMAR et al., 2012;2013). Após vasta revisão de literatura, Pothuraju et al. (2014) concluíram que diferentes extratos desta espécie tem atividade na prevenção de ganho de peso, na regulação da glicose plasmática e na redução da acumulação de lipídeos no epidídimo e fígado. Além disso, vários trabalhos indicam seu potencial hipoglicemiante e hipolimpêmico (AL-ROMAIYAN et al., 2013; PRABHAKAR; DOBLE, 2011; PRABHU; VIJAYAKUMAR, 2014; YOGALAKSHMI; VAIDEHI; RAMAKOTTI, 2014).

Embora a maioria dos fitoterápicos citados estejam sendo utilizados para a finalidade correta, como demonstrado acima, o baixo número de pacientes que usam com base em prescrição, apenas $5 \%$, e que informam ao médico, apenas $8 \%$, é muito preocupante. Ainda que, em geral, os fitoterápicos representem menor risco à saúde do que o uso de substâncias isoladas, como no caso dos medicamentos sintéticos e fitofármacos, o uso da dosagem incorreta, a associação de mais de uma espécie e o uso concomitante com outros medicamentos podem trazer grandes riscos à saúde, devendo ser criticamente avaliado (CHIBA et al., 2015; DE PINHO et al., 2010).

Exemplo de associação, que feita com dosagens erradas pode trazer complicações, é a combinação de $C$. sinensis e $P$. cupana, pois ambos possuem cafeína. Em pequenas doses a cafeína não apresenta um perigo para a saúde, mas quando utilizada em excesso pode trazer problemas como aumentar o risco de rabdomiólise por esforço (CAROL, 2013). Existem relatos de casos de pacientes que utilizaram O. biennis concomitantemente com anestésicos ou flufenazina e clorpromazina e apresentaram convulsões devido à redução do limiar convulsão (JOHNE; ROOTS, 2005). Outro aspecto que deve ser abordado é influência na farmacocinética. C. sinensis reduz a expressão de lipoproteína-P e interfere em algumas enzimas da superfamília citocromo $\mathrm{P} 450$, podendo refletir diretamente na capacidade de metabolização de vários grupos de fármacos $(\mathrm{CHO} ; \mathrm{YOON}, 2015)$.

Como observado por Barreto e Silveira (2014), a maioria dos cursos de Nutrição e Medicina das Instituições de Ensino Superior do Brasil não apresenta disciplinas obrigatórias sobre plantas medicinais e fitoterápicos e poucas apresentam disciplinas eletivas. Desta forma, a indiferença dos médicos quanto à utilização de fitoterápicos, relatada pelos pacientes, encontrada neste estudo pode estar relacionada com a 
falta de capacitação sobre o assunto. Mesmo com a falta de disciplinas específicas, o fato de os médicos e nutricionistas apresentarem conhecimentos sobre fisiologia, bioquímica e farmacologia dá a estes profissionais uma boa base para cursos de aperfeiçoamento e/ou especialização. Neste contexto, a legislação vigente representa a realidade dos profissionais de saúde brasileiros no que tange a prescrição de plantas medicinais e fitoterápicos (CFM, 1992; CFN, 2013). A formação que apresenta melhor preparação neste contexto é em de Farmácia, onde a maioria dos cursos apresentam disciplinas obrigatórias e eletivas (BARRETO; SILVEIRA, 2014). Desta forma, a legislação do Conselho Federal de Farmácia também representa a realidade dos profissionais farmacêuticos no contexto das plantas medicinais e fitoterápicos (CFF, 2011). Deve-se destacar, porém, que embora a legislação represente os profissionais de saúde, ela não tem atingido seu maior objetivo que é trazer segurança para os usuários, uma vez foi demonstrado nesse estudo que $90 \%$ dos usuários de fitoterápicos nas academias de musculação utilizam os ergogênicos sem orientação profissional.

\section{CONCLUSÃO}

É possível concluir que a utilização de fitoterápicos com propósitos ergogênicos é uma prática corriqueira entre os praticantes da modalidade musculação das academias onde este trabalho foi realizado, porém na maioria das vezes não é praticada com o respaldo de profissionais balizados pela legislação e conhecimento científico adequado, configurando uma situação de risco aos usuários. Desta forma é necessária maior interação entre os profissionais prescitores e usuários visando o cumprimento da legislação vigente e consequentemente o uso seguro de plantas medicinais e fitoterápicos.

\section{REFERÊNCIAS}

AHMED, H.H.; MANNAA, F.; EL-SAYED, E.M. Ameliorative effect of artichoke (Cynara scolymus L.) extracts on creatine monohydrate-induced renal dysfunction in male rats. Deutsche Lebensmittel-Rundschau, v. 104, n. 1, p. 29-36, Jan 2008.

AL-ROMAIYAN, A.; KING, A.J.; PERSAUD, S.J.; JONES, P.M. A Novel Extract of Gymnema sylvestre Improves Glucose Tolerance In Vivo and Stimulates Insulin Secretion and Synthesis In Vitro. Phytotherapy Research, v. 27, n. 7, p. 1006-1011, 2013.

ALEXÍADES, M. Selected guidelines for ethnobotanical research: a field manual. New York: The New York Botanical Garden, 1996.

ANTONIO, J.; UELMEN, J.; RODRIGUEZ, R.; EARNEST, C. The effects of Tribulus terrestris on body composition and exercise performance in resistance-trained males. Int J Sport Nutr Exerc Metab, v. 10, n. 2, p. 208-15, Jun 2000.

BARRETO, B.B.; SILVEIRA, D. Inclusion of courses on phytotherapy in undergraduate curriculum of health-related courses. Journal of Medicinal Plants Research, v. 8, n. 47, p. 1374-1386, 2014.

BARRETO, S.M.G. Esporte e Saúde. Revista Eletrônica de Ciências, v. 22, n. 4, 2003.

BENT, S.; PADUlA, A.; NEUHAUS, J. Safety and efficacy of Citrus aurantium for weight loss. The American Journal of Cardiology, v. 94, n. 10, p. 1359-1361, 11/15/ 2004.

BROWN, G.A.; VUKOVICH, M.D.; MARTINI, E.R.; KOHUT, M.L.; FRANKE, W.D.; JACKSON, D.A.; KING, D.S. Effects of androstenedione-herbal supplementation on serum sex hormone concentrations in 30to 59-year-old men. Int J Vitam Nutr Res, v. 71, n. 5, p. 293-301, Sep 2001. 
BROWN, G.A.; VUKOVICH, M.D.; REIFENRATH, T.A.; UHL, N.L.; PARSONS, K.A.; SHARP, R.L.; KING, D.S. Effects of anabolic precursors on serum testosterone concentrations and adaptations to resistance training in young men. Int J Sport Nutr Exerc Metab, v. 10, n. 3, p. 340-59, Sep 2000.

CAROL, M.L. Hydroxycut weight loss dietary supplements: a contributing factor in the development of exertional rhabdomyolysis in three U.S. Army soldiers. Mil Med, v. 178, n. 9, p. e1039-42, Sep 2013.

CASTRO, A.L. Cultura contemporânea, identidades e sociabilidades: olhares sobre o corpo, mídia e novas tecnologias. São Paulo: UNESP, 2010.

CAVICHIOLI, B.; ABOURIHAN, C.L.S.; PASSONI, C.M.S. Monitoramento da administração de um suplemento como coadjuvante na perda de peso. Cadernos das Escolas de Saúde, v. 6, p. 90-110, 2012.

CHIBA, T.; SATO, Y.; SUZUKI, S.; UMEGAKI, K. Concomitant Use of Dietary Supplements and Medicines in Patients due to Miscommunication with Physicians in Japan. Nutrients, v. 7, n. 4, p. 2947-2960, 2015.

CHO, H.J.; YOON, I.S. Pharmacokinetic interactions of herbs with cytochrome p450 and p-glycoprotein. Evid Based Complement Alternat Med, v. 2015, p. 736431, 2015.

CIFUENTE, D.A.; GIANELlO, J.C.; MARIA, A.O.M.; PETENATTI, E.M.; PETENATTI, M.M.; DEL VITTO, L.A.; TONN, C.E. Choleretic Activity of Five Species of Baccharis ("Carquejas") Used as Phytotherapics in Argentinean Traditional Medicine. Latin American Journal of Pharmacy, v. 29, n. 6, p. 1053-1056, Sep 2010.

CONSELHO FEDERAL DE FARMÁCIA. Resolução n. 546 de 21 de julho de 2011. Dispõe sobre a indicação farmacêutica de plantas medicinais e fitoterápicos isentos de prescrição e o seu registro. Relator Senador Demóstenes Torres. Diário Oficial da República Federativa do Brasil, Brasília, 26 de jul. 2011. Sec. 1, p. 87

CONSELHO FEDERAL DE MEDICINA. Parecer n. 04 de 15 de janeiro de 1992. Reconhece a acupuntura e a fitoterapia como métodos terapêuticos. Relator Cons. Nilo Fernando Rezende Vieira. Diário Oficial da Reública Federativa do Brasil, Brasília, 1992.

CONSELHO FEDERAL DE NUTRICIONISTAS. Resolução n. 525 de 25 de junho de 2013. Regulamenta a prática da fitoterapia pelo nutricionista, atribuindo-lhe competência para, nas modalidades que especifica, prescrever plantas medicinais, drogas vegetais e fitoterápicos como complemento da prescrição dietética e, dá outras providências. Diário Oficial da República Federativa do Brasil, Brasília, 28 de jun. 2013. Sec. 1, p. 141

COSTA, E.M.B.; VENÂNCIO, S. Atividade física e saúde: Discursos que controlam o corpo. Revista Pensar a Pratica, v. 7, n. 1, p. 59-74, 2004.

DE PINHO, D.S.; STURBELLE, R.T.; MARTINO-ROTH, M.G.; GARCIAS, G.L. Evaluation of mutagenic activity resulting from the infusion baccharis trimera (Less.) DC. using the allium cepa test and a chromosomal test for aberrations in human lymphocytes. Brazilian Journal of Pharmacognosy, v. 20, n. 2, p. 165-170, 2010.

ESPINOLA, E.B.; DIAS, R.F.; MATTEI, R.; CARLINI, E.A. Pharmacological activity of Guarana (Paullinia cupana Mart.) in laboratory animals. J Ethnopharmacol, v. 55, n. 3, p. 223-9, Feb 1997. 
FOX, E.L.; BOWERS, R.W.; FOSS, M.L. The physiological basis for exercise and sport. Madison: Brown and Benchmark, 1988.

GRANCE, S.R.; TEIXEIRA, M.A.; LEITE, R.S.; GUIMARAES, E.B.; DE SIQUEIRA, J.M.; DE OLIVEIRA FILIU, W.F.; DE SOUZA VASCONCELOS, S.B.; DO CARMO VIEIRA, M. Baccharis trimera: effect on hematological and biochemical parameters and hepatorenal evaluation in pregnant rats. J Ethnopharmacol, v. 117, n. 1, p. 28-33, Apr 172008.

GRAY, S.; WOOLF, A.D. Citrus aurantium used for weight loss by an adolescent with anorexia nervosa. Journal of Adolescent Health, v. 37, n. 5, p. 414-415, 11// 2005.

GUPTA, H.; PAWAR, D.; RIVA, A.; BOMBARDELlI, E.; MORAZZONI, P. A Randomized, Doubleblind, Placebo-controlled Trial to Evaluate Efficacy and Tolerability of an Optimized Botanical Combination in the Management of Patients with Primary Hypercholesterolemia and Mixed Dyslipidemia. Phytotherapy Research, v. 26, n. 2, p. 265-272, Feb 2012.

HANSEN, D.K.; GEORGE, N.I.; WHITE, G.E.; PELLICORE, L.S.; ABDEL-RAHMAN, A.; FABRICANT, D. Physiological effects following administration of Citrus aurantium for 28 days in rats. Toxicology and Applied Pharmacology, v. 261, n. 3, p. 236-247, 6/15/ 2012.

HONG, N.-Y.; CUI, Z.-G.; KANG, H.-K.; LEE, D.-H.; LEE, Y.-K.; PARK, D.-B. p-Synephrine stimulates glucose consumption via AMPK in L6 skeletal muscle cells. Biochemical and Biophysical Research Communications, v. 418, n. 4, p. 720-724, Feb 242012.

JOHNE, A.; ROOTS, I. Clinical Drug Interactions with Medicinal Herbs. Evidence-Based Integrative Medicine, v. 2, n. 4, p. 207-228, 2005/12/01 2005.

KENNY, W.L.; WILMORE, J.H.; COSTILL, D.L. Physiology of sport and exercise. Champaign: Human Kinects, 2011.

KLEIN, T.; LONGHINI, R.; BRUSCHI, M.L.; MELLO, J.C.P. Fitoterápicos: um mercado promissor. Revista de Ciências Farmacêuticas Básica e Aplicada, v. 30, n. 3, p. 241-248, 2009.

KUMAR, V.; BHANDARI, U.; TRIPATHI, C.D.; KHANNA, G. Evaluation of antiobesity and cardioprotective effect of Gymnema sylvestre extract in murine model. Indian Journal of Pharmacology, v. 44, n. 5, p. 607-613, Sep-Oct 2012.

. Anti-obesity effect of Gymnema sylvestre extract on high fat diet-induced obesity in Wistar rats. Drug Res (Stuttg), v. 63, n. 12, p. 625-32, Dec 2013.

LEE, S.; PITESA, M.; PILLUTLA, M.; THAU, S. When beauty helps and when it hurts: An organizational context model of attractiveness discrimination in selection decisions. Organizational Behavior and Human Decision Processes, v. 128, n. 0, p. 15-28, 5// 2015.

LIMA, W.P.; CARNEVALI, L.C., JR.; EDER, R.; COSTA ROSA, L.F.; BACCHI, E.M.; SEELAENDER, M.C. Lipid metabolism in trained rats: effect of guarana (Paullinia cupana Mart.) supplementation. Clin Nutr, v. 24, n. 6, p. 1019-28, Dec 2005.

LOI, B.; FANTINI, N.; COLOMBO, G.; GESSA, G.L.; RIVA, A.; BOMBARDELLI, E.; MORAZZONI, P.; CARAI, M.A.M. Reducing Effect of a Combination of Phaseolus vulgaris and Cynara scolymus Extracts on Food Intake and Glycemia in Rats. Phytotherapy Research, v. 27, n. 2, p. 258-263, 2013 a. 
Reducing effect of an extract of Phaseolus vulgaris on food intake in mice - Focus on highly palatable foods. Fitoterapia, v. 85, n. 0, p. 14-19, 3// $2013 \mathrm{~b}$.

LU, C.H.; YANG, T.H.; WU, C.C.; DOONG, J.Y.; LIN, P.Y.; CHIANG, C.M.; LIN, C.L.; HSIEH, S.L. Clinical evaluation of Garcinia cambogia and Phaseolus vulgaris extract for obese adults in taiwan. Nutritional Sciences Journal, v. 37, n. 2, p. 75-84, 2012.

MAIOLI, H.N. Avaliação do consumo de suplementos por mulheres praticantes de atividade física em uma academia na cidade de taguatinga-DF. Revista Brasileira de Nutrição Esportiva, v. 6, n. 32, p. 118-125, 2012.

MILASIUS, K.; DADELIENE, R.; SKERNEVICIUS, J. The influence of the Tribulus terrestris extract on the parameters of the functional preparedness and athletes' organism homeostasis. Fiziol Zh, v. 55, n. 5, p. 89-96, 2009.

OLIVEIRA, A.C.; ENDRINGER, D.C.; AMORIM, L.A.; DAS GRACAS, L.B.M.; COELHO, M.M. Effect of the extracts and fractions of Baccharis trimera and Syzygium cumini on glycaemia of diabetic and nondiabetic mice. J Ethnopharmacol, v. 102, n. 3, p. 465-9, Dec 12005.

ORGANIZACIÓN MUNDIAL DE LA SALUD. Estrategia de la OMS sobre medicina tradicional 20022005. Genebra, 67 p., 2002.

PENDLETON, M.; BROWN, S.; THOMAS, C.; ODLE, B. Potential Toxicity of Caffeine When Used as a Dietary Supplement for Weight Loss. Journal of Dietary Supplements, v. 9, n. 4, p. 293-298, 2012.

POTHURAJU, R.; SHARMA, R.K.; CHAGALAMARRI, J.; JANGRA, S.; KUMAR KAVADI, P. A systematic review of Gymnema sylvestre in obesity and diabetes management. Journal of the Science of Food and Agriculture, v. 94, n. 5, p. 834-840, 2014.

PRABHAKAR, P.; DOBLE, M. Mechanism of action of natural products used in the treatment of diabetes mellitus. Chinese Journal of Integrative Medicine, v. 17, n. 8, p. 563-574, 2011/08/01 2011.

PRABHU, S.; VIJAYAKUMAR, S. Antidiabetic, hypolipidemic and histopathological analysis of Gymnema sylvestre ( $\mathrm{R} . \mathrm{Br}$ ) leaves extract on streptozotocin induced diabetic rats. Biomedicine \& Preventive Nutrition, v. 4, n. 3, p. 425-430, 7// 2014.

RATES, S.M.K. Promoção do uso racional de fitoterápicos: uma abordagem no ensino de Farmacognosia. Revista Brasileira De Farmacognosia, v. 11, n. 2, p. 57-69, 2001.

ROGERSON, S.; RICHES, C.J.; JENNINGS, C.; WEATHERBY, R.P.; MEIR, R.A.; MARSHALLGRADISNIK, S.M. The effect of five weeks of Tribulus terrestris supplementation on muscle strength and body composition during preseason training in elite rugby league players. J Strength Cond Res, v. 21, n. 2, p. 348-53, May 2007.

RONDANELlI, M.; GIACOSA, A.; ORSINI, F.; OPIZZI, A.; VILLANI, S. Appetite Control and Glycaemia Reduction in Overweight Subjects treated with a Combination of Two Highly Standardized Extracts from Phaseolus vulgaris and Cynara scolymus. Phytotherapy Research, v. 25, n. 9, p. 1275-1282, 2011. 
RYAN, M.; LAZAR, I.; NADASDY, G.M.; NADASDY, T.; SATOSKAR, A.A. Acute kidney injury and hyperbilirubinemia in a young male after ingestion of Tribulus terrestris. Clin Nephrol, v. 83, n. 3, p. 177-83, Mar 2015.

SAE-TAN, S.; ROGERS, C.J.; LAMBERT, J.D. Decaffeinated Green Tea and Voluntary Exercise Induce Gene Changes Related to Beige Adipocyte Formation in High Fat-Fed Obese Mice. J Funct Foods, v. 14, p. 210-214, Apr 12015.

SANTOS, M.A.A.; SANTOS, R.P. Uso de suplementos alimentares como forma de melhorar a performance nos programas de atividade física em academias de ginástica. Revista Paulista de Educação física, v. 16, n. 2, p. 174-185, 2002.

SCHMITT, G.C.; ARBO, M.D.; LORENSI, A.L.; MACIEL, E.S.; KRAHN, C.L.; MARIOTTI, K.C.; DALLEGRAVE, E.; LEAL, M.B.; LIMBERGER, R.P. Toxicological Effects of a Mixture Used in Weight Loss Products: p-Synephrine Associated With Ephedrine, Salicin, and Caffeine. International Journal of Toxicology, v. 31, n. 2, p. 184-191, Mar-Apr 2012.

SILVESTRINI, G.I.; MARINO, F.; COSENTINO, M. Effects of a commercial product containing guarana on psychological well-being, anxiety and mood: a single-blind, placebo-controlled study in healthy subjects. J Negat Results Biomed, v. 12, p. 9, 2013.

SOUZA, S.P.I.; PEREIRA, L.L.S.; SOUZA, A.A.; DOS SANTOS, C.D. Inhibition of pancreatic lipase by extracts of Baccharis trimera: evaluation of antinutrients and effect on glycosidases. Brazilian Journal of Pharmacognosy, v. 21, n. 3, p. 450-455, May-Jun 2011.

SOUZA, S.P.I.; PEREIRA, L.L.S.; SOUZA, A.A.; SANTOS, C.D. Selection of crude plant extracts with anti-obesity activity. Revista Brasileira de Plantas Medicinais, v. 14, n. 4, p. 643-648, 2012.

STOHS, S.J.; PREUSS, H.G.; SHARA, M. A Review of the Human Clinical Studies Involving Citrus aurantium (Bitter Orange) Extract and its Primary Protoalkaloid p-Synephrine. International Journal of Medical Sciences, v. 9, n. 7, p. 527-538, 2012.

TIRAPEGUI, J. Nutrição, metabolismo e suplementação na prática esportiva. São Paulo: Atheneu, 2005.

TORRES-FUENTES, C.; SCHELLEKENS, H.; DINAN, T.G.; CRYAN, J.F. A natural solution for obesity: bioactives for the prevention and treatment of weight gain. A review. Nutr Neurosci, v. 18, n. 2, p. 49-65, Feb 2015.

VALENZUELA, A.B.A. El consumo te y la salud: caraterísticas y propiedades beneficas de esta bebida milenaria. Revista Chileña de Nutricion, v. 31, n. 2, p. 72-82, 2004.

XU, Y.; ZHANG, M.; WU, T.; DAI, S.; XU, J.; ZHOU, Z. The anti-obesity effect of green tea polysaccharides, polyphenols and caffeine in rats fed with a high-fat diet. Food Funct, v. 6, n. 1, p. 297-304, Jan 2015.

YANG, X.; YIN, L.; LI, T.; CHEN, Z. Green tea extracts reduce adipogenesis by decreasing expression of transcription factors C/EBPalpha and PPARgamma. Int J Clin Exp Med, v. 7, n. 12, p. 4906-14, 2014.

YOGALAKSHMI, K.; VAIDEHI, J.; RAMAKOTTI, P. Hypoglycemic effect of Gymnema sylvestre leaf extract on normal and streptozotocin induced diabetic rats. International Journal of ChemTech Research, $v$. 6, n. 12, p. 5146-5150, 2014. 
YUNES, R.A.; PEDROSA, R.C.; FILHO, V.C. Fármacos e fitoterápicos: a necessidade do desenvolvimento da indústria de fitoterápicos e fitofármacos no Brasil. Química Nova, v. 24, n. 1, p. 147-152, 2001.

ZHU, Z.J.; JIANG, W.Q.; THOMPSON, H.J. Edible dry bean consumption (Phaseolus vulgaris L.) modulates cardiovascular risk factors and diet-induced obesity in rats and mice. British Journal of Nutrition, v. 108, p. S66-S73, Aug 2012. 